\title{
Evaluation of Transabdominal and Transperineal Ultrasound-Derived Prostate Specific Antigen (PSA) Density and Clinical Utility Compared to MRI Prostate Volumes: A Feasibility Study
}

\author{
Maria Pantelidou \\ University of Cambridge \\ Iztok Caglic \\ University of Cambridge \\ Anne George \\ University of Cambridge \\ Oleg Blyuss \\ University of Hertfordshire \\ Vincent Gnanapragasam \\ University of Cambridge
}

Tristan Barrett ( $\nabla$ tristan.barrett@gmail.com )

University of Cambridge

\section{Research Article}

Keywords: ultrasound-derived PSA-density, prostate cancer, Intra-class correlation coefficient, MRI

Posted Date: November 30th, 2021

DOl: https://doi.org/10.21203/rs.3.rs-1083049/v1

License: (a) (1) This work is licensed under a Creative Commons Attribution 4.0 International License. Read Full License 


\section{Abstract}

\section{Purpose}

To investigate the accuracy of surface-based ultrasound-derived PSA-density (US-PSAD) versus gold-standard MRI-PSAD as a risk-stratification tool.

\section{Methods}

Single-centre prospective study of patients undergoing MRI for suspected prostate cancer (PCa). Four combinations of US-volumes were calculated using transperineal (TP) and transabdominal (TA) views, with triplanar measurements to calculate volume and US-PSAD. Intra-class correlation coefficient (ICC) was used to compare US and MRI volumes. Categorical comparison of MRI-PSAD and US-PSAD was performed at PSAD cutoffs $<0.15,0.15-0.20$, and $>0.20 \mathrm{ng} / \mathrm{mL}^{2}$ to assess agreement with MRI-PSAD risk-stratification decisions.

\section{Results}

64 men were investigated, mean age 69 years and PSA $7.0 \mathrm{ng} / \mathrm{mL}$. 36/64 had biopsy-confirmed prostate cancer (18 Gleason 3+3, 18 Gleason $\geq 3+4$ ). Mean MRI-derived gland volume was $60 \mathrm{~mL}$, compared to $56 \mathrm{~mL}$ for TA-US, and $65 \mathrm{~mL}$ TP-US. ICC demonstrated good agreement for all US volumes with MRI, with highest agreement for transabdominal US, followed by combined TA/TP volumes. Risk-stratification decisions to biopsy showed concordant agreement between triplanar MRI-PSAD and ultrasound-PSAD in $86-91 \%$ and $92-95 \%$ at PSAD thresholds of $>0.15 \mathrm{ng} / \mathrm{mL}^{2}$ and $>0.12 \mathrm{ng} / \mathrm{mL}^{2}$, respectively. Biopsy-decision making at threshold $>0.12 \mathrm{ng} / \mathrm{mL}^{2}$, demonstrated sensitivity ranges of $81-100 \%$, specificity $85-100 \%$, PPV $86-100 \%$ and NPV $83-100 \%$. Transabdominal US provided optimal sensitivity of $100 \%$ for this clinical decision, with specificity $85 \%$, and transperineal US provided optimal specificity of $100 \%$, with sensitivity $87 \%$.

\section{Conclusion}

Transperineal-US and combined TA-TP US-derived PSA density values compare well with standard MRI-derived values and could be used to provide accurate PSAD at presentation and inform the need for further investigations.

\section{Key Points}

1. Good inter-reader agreement was demonstrated between MRI and US-derived prostate volumes

2. US and MRI agreement ranged between $86-91 \%$ at a PSA density threshold of $0.15 \mathrm{ng} / \mathrm{mL}^{2}$ and $92-95 \%$ at a threshold of $>0.12 \mathrm{ng} / \mathrm{mL}^{2}$

3. At a US PSA density-derived biopsy threshold of $>0.12 \mathrm{ng} / \mathrm{mL}^{2}$, transabdominal views provide highest sensitivity (100\%)

4. Transperineal views provide more accurate sagittal measurements, being independent of bladder filling and body habitus

\section{Introduction}


Prostate cancer ( $\mathrm{PCa}$ ) accounts for almost 1-in-5 of all new male cancer diagnoses [1], with the incidence of the disease expected to double by 2030 in part due to the ageing population [2]. The adoption of prostate-specific antigen (PSA) test for screening symptomatic patients in the mid-1990s dramatically changed the profile of PCa patients, with trends towards detection of lower grade disease, including clinically insignificant tumours [3]. A raised PSA level only has a $30 \%$ positive predictive value for cancer [4] and is more commonly associated with benign prostatic hypertrophy (BPH), conversely, 37\% of men with a PSA reading in the normal range of 2.5-4 $\mathrm{ng} / \mathrm{ml}$ still harbour prostate cancer [5], thus PSA screening is not routinely recommended [6]. Prostate multiparametric (mp) MRI can aid the diagnostic process by avoiding biopsy when negative in low-risk patients and in directing biopsy to suspicious targets when positive, and is now recommended within international and European guidelines as the initial diagnostic step for men with suspected PCa $[7,8]$.

MRI has a high negative predictive value for the presence of clinically significant (cs) PCa and can help avoid biopsy in 27-49\% of patients [9-12]. However, MRI is an expensive resource and there is increasing pressure on MRI services to provide rapid access to MRI and prompt reporting times, in the face of ever increasing demand [13]. Given that a high proportion of mpMRI studies are negative, a triage step that could safely avoid such imaging in a proportion of patients would be desirable. PSA-density based on MRI-derived prostate volumes has been shown to be a more reliable biomarker than PSA alone [14-16]. In addition, PSA-density is now considered an integral part of the biopsy decision making process, with a recent consensus paper stating that PSA-density should be used to help avoid biopsy when MRI is negative and to augment the decision-making process when MRI findings are equivocal [17]. However, prostate gland volume can also be derived from other imaging modalities including ultrasound (US) which, although operator-dependent, is relatively inexpensive, quick to perform, and widely available. To date, however, most studies comparing US with MRI have employed transrectal ultrasound which while is invasive and intrusive for patients. A more acceptable alternative is surface-based US with images acquired via either the abdomen or perineum.

The aim of this study was therefore to investigate how well surface-based US-derived PSA-density performs in comparison to the gold-standard of MRI-derived PSA-density and whether it could be used in clinical practice as a risk-stratification and triage tool for deciding whether patients require urgent MR imaging or could safely avoid further investigations.

\section{Methods}

This was a single tertiary centre prospective cohort feasibility study, with written informed consent obtained from all participants. All elements of this prospective study were carried out in accordance with the Declaration of Helsinki and were approved by the institutional ethics board (NRES Committee East of England, UK, ref: NRES 03/018), with written informed consent obtained from all participants. All methods were performed in accordance with the relevant guidelines and regulations. The inclusion criteria for the study were men having a prostate MRI for suspected prostate cancer and subsequently undergoing prostatic biopsy, regardless of whether cancer was present on biopsy. Exclusion criteria were a clinical diagnosis of prostatitis and/or abscess, presence of hip metalwork, and/or any previous treatment for prostate cancer. 65 participants were recruited, with one patient excluded due to loss of imaging data transfer to PACS. The primary outcome of the study was to compare US-derived prostate volume with MRI prostate volume and thus determine the accuracy of surfacebased US-derived PSA-density values. The secondary outcome was to derive relevant US PSA-density cut-off values that could be applied in clinical practice to predict the need for biopsy and/or presence of significant PCa. 


\section{Ultrasound Imaging:}

All scans were performed on a standard commercially available ultrasound machine (Toshiba Aplio XG), using a low frequency curvilinear abdominal transducer. All patients were scanned with a full-bladder by a single, experienced uro-radiologist. Two standard ultrasound views were performed: trans-perineal (TP) and transabdominal (TA). For each view, three orthogonal measurements were acquired and used to calculated the USderived prostatic volume: right-to-left (axial 1), antero-posterior (axial 2) and cranio-caudal (sagittal) (Figure 1).

\section{MRI Imaging:}

3T-MRI (MR750, GE Healthcare) was performed for all participants using a 32-channel phased-array coil. The following sequences were acquired: T2-weighted 2D fast recovery FSE images in axial, sagittal and coronal planes and sagittal T2 3D FSE sequences, axial T1-weighted imaging: TR/TE 561/11 ms, FOV $24 \times 24 \mathrm{~cm}$, resolution $1.1 \times 1.0 \mathrm{~mm}$; axial diffusion-weighted imaging (DWI) dual spin echo-planar imaging and axial 3D dynamic contrast-enhanced (DCE) imaging following a bolus of Gadobutrol (Gadovist, Bayer). The T2-weighted imaging was used to derive prostate volume data.

\section{Imaging analysis:}

Four different US-derived prostatic gland volumes were derived, using transabdominal (TA) and transperineal (TP) views: US-TA volume, US-TP volume, and using two combinations of TA and TP volumes, US-TA-TP volume and US-TP-TA, respectively, to overcome shadowing from the pubis bone (Figure 2). US TA-TP was derived using the Axial 1 and Axial 2 measurements of derived from transabdominal views and combined with the sagittal measurement acquired transperineally (Table 1). US-TP-TA was derived using the axial 1 transabdominal measurement combined with the Axial 2 and sagittal measurements acquired transperineally. MRI volume was calculated using triplanar measurements derived from the axial and sagittal T2-weighted sequences, following the PI-RADS guidelines [17]. MRI volume was additionally calculated using whole gland segmentation software (DynaCAD Prostate ${ }^{T w}$, Philips). All US and MRI-derived measurements were performed by two experienced uroradiologists independently, and blinded to any clinical details.

Table 1

US views acquired for deriving prostate volumes

\begin{tabular}{|llll|}
\hline View & Axial 1 (R-L) & Axial 2 (A-P) & Cranio-caudal \\
\hline TA US & Transabdominal & Transabdominal & Transabdominal \\
\hline TP US & Transperineal & Transperineal & Transperineal \\
\hline TA/TP & Transabdominal & Transabdominal & Transperineal \\
\hline TP-TA US & Transabdominal & Transperineal & Transperineal \\
\hline
\end{tabular}

\section{Statistics:}

A two-way mixed effects model intra-class correlation coefficient (ICC) was used to measure how closely the US derived prostate volumes (TA, TP, TA/TP and TP-TA) compared to the gold-standard triplanar MRI-derived prostate volume and to assess inter-rater agreement [18]. ICC values were defined as indicating poor $(<0.5)$, moderate (0.5-0.75), good (0.75-0.90) and excellent reliability (>0.90) [19]. We considered any cases with more than $50 \%$ percentage difference between the MRI- and any of the US- derived prostate volumes as outliers. 
Categorical comparison was made between MRI PSA-density (MRI-PSAD) and each US-PSAD at three PSAD thresholds based on prior literature $[14,17,20]:<0.15,0.15-0.20,>0.20 \mathrm{ng} / \mathrm{mL}^{2}$ in order to ascertain whether each US-PSAD was in agreement with the MRI-PSAD decision on risk stratification. In addition, a PSA-Categorical comparison was made between the MRI PSA-density and each of the four US derived PSA-densities at these PSAD cut-off values in order to ascertain whether each US-derived PSA density would be in agreement with the MRI derived PSA-density and the theoretical decision of whether to perform biopsy or not.

\section{Results}

64 patients completed the study, with a mean age of 69 years (IQR: 64-73), mean PSA of $7.0 \mathrm{ng} / \mathrm{mL}$ (IQR: 5-9 $\mathrm{ng} / \mathrm{mL}$ ), and mean BMI of 27 (IQR: 24-29); Table 2. 36/64 (56.3\%) patients had biopsy-confirmed prostate cancer: 18 Grade Group 1, 15 Grade Group 2, and three Grade Group $\geq 3$.

Table 2

Patient Demographics

\begin{tabular}{|ll|}
\hline Characteristic & \\
\hline Patient age, years (IQR) & $69(64-73)$ \\
\hline PSA level, ng/mL (IQR) & $7.0(5.0-9.0)$ \\
\hline BMI (IQR) & $27.0(24.0-29.0)$ \\
\hline Biopsy outcome & \\
\hline Negative & $28(43.8 \%)$ \\
\hline Gleason $3+3$ & $18(28.1 \%)$ \\
\hline Gleason $3+4$ & $15(23.4 \%)$ \\
\hline Gleason $\geq 4+3$ & $3(4.7 \%)$ \\
\hline
\end{tabular}

\section{Prostate volumetric analysis}

The mean volumetric segmentation-derived MRI prostate volume was $60 \mathrm{~mL}$ (IQR: 40-76 mL). The mean triplanar MRI-derived prostate volume was $61 \mathrm{~mL}$ (IQR: 39-79 mL). The average US derived volumes were $56 \mathrm{~mL}$ (IQR: 34$75 \mathrm{~mL})$ for TA, $65 \mathrm{~mL}(46-71 \mathrm{~mL})$ for TP, $66 \mathrm{~mL}(42-82 \mathrm{~mL})$ for TA/TP, and $67 \mathrm{~mL}$ (46-80 mL) for TA-TP.

\section{ICC agreement between US and MRI-derived gland volumes}

ICC revealed good agreement between radiologist-measured, clinical standard triplanar MRI-volume versus the gold-standard volumetric derived segmentation-derived MRI volumes (ICC 0.965; Table 3). Good agreement was demonstrated between all US derived prostate volumes and the gold-standard MRI-derived volumes calculated using 3-plane measurements, with highest ICC for US TA and US TA/TP Volume at 0.873 (95\% Cl: 0.798-0.921) and 0.874 (95\% Cl: 0.755-0.931), respectively. The lowest level of agreement was demonstrated for US TP Volume at 0.776 (95\% Cl: 0.654-0.858) (Table 3). A maximum of 3/64 cases lay outside the $95 \%$ confidence interval (Cl) of agreement lines. 
Table 3

Intraclass correlation coefficient (ICC) agreement between MRIderived and US-derived prostate volumes.

\begin{tabular}{|ll|}
\hline Analysis & ICC (95\% CI) \\
\hline 3-plane MRI vs MRI Volumetric (Dynacad) & $0.965(0.942-0.978)$ \\
\hline 3-plane MRI vs US TA Volume & $0.873(0.798-0.921)$ \\
\hline 3-plane MRI vs US TP Volume & $0.776(0.654-0.858)$ \\
\hline 3-plane MRI vs US TA/TP Volume & $0.874(0.755-0.931)$ \\
\hline 3-plane MRI vs TA-TP Volume & $0.847(0.709-0.915)$ \\
\hline
\end{tabular}

\section{ICC inter-rater agreement}

Excellent inter-reader agreement was shown for readers' MRI and US-derived prostate volumes, with the greatest agreement noted between US-TA/TP and US TA volumes at 0.977 (95\% Cl: 0.959-0.987) and 0.963 (0.916-0.981), respectively (Table 4). 6/64 cases showed $\geq 50 \%$ percentage difference between the MRI-derived volume and at least one of the US-derived calculations and could be considered as relative outliers. Out of these, two patients had a BMI of $>30$, with one further patient noted to have a defect due to a previous transurethral resection of the prostate (TURP) procedure, limiting the accuracy of the segmentation-derived volume (Figures 3,4 ).

Table 4

Intraclass correlation coefficient (ICC) interobserver agreement for MRI- and US- derived prostate volumes.

\begin{tabular}{|ll|}
\hline Analysis & ICC $(95 \%$ CI) \\
\hline 3-plane MRI Volume & $0.984(0.974-0.99)$ \\
\hline US TA Volume & $0.963(0.916-0.981)$ \\
\hline US TP Volume & $0.903(0.84-0.941)$ \\
\hline US TA/TP Volume & $0.977(0.959-0.987)$ \\
\hline US TA-TP Volume & $0.913(0.856-0.947)$ \\
\hline
\end{tabular}

\section{Categorical comparison between MRI and US derived PSA- density}

When evaluating the three PSA-density categories of $<0.15 \mathrm{ng} / \mathrm{mL}^{2}, 0.15-0.20 \mathrm{ng} / \mathrm{mL}^{2}$ and $>0.20 \mathrm{ng} / \mathrm{mL}^{2}$, agreement was demonstrated between triplanar-derived MRI-PSAD and US-PSAD in $83-88 \%$ of cases, with highest agreement for TA/TP US-PSAD (Table 5). Simplifying this to a decision to biopsy or not at a PSA-density threshold of $>0.15 \mathrm{ng} / \mathrm{mL}^{2}$, US PSAD matched the MR decision in 86-91\% of cases with the TP-TA US-PSAD performing best. Given that the ultrasound-derived measurements tended to over-estimate prostatic volume, we applied a lower, and clinically more conservative threshold of $0.12 \mathrm{ng} / \mathrm{mL}^{2}$ for a decision to proceed to biopsy. This resulted in an increased agreement with the MRI-equivalent decision of $92-95 \%$ of cases with highest agreement for transabdominal US-PSAD (Table 5). 
Table 5

Categorical comparison of MRI and US-derived PSA-density (PSA-D) at different biopsy thresholds of $<0.15$ $\mathrm{ng} / \mathrm{mL}^{2}, 0.15-0.20 \mathrm{ng} / \mathrm{mL}^{2}$ and $>0.20 \mathrm{ng} / \mathrm{mL}^{2}$ ) and agreement to biopsy or not at PSA-density thresholds of 0.15 $\mathrm{ng} / \mathrm{mL}^{2}$ and $0.12 \mathrm{ng} / \mathrm{mL}^{2}$

\begin{tabular}{|llll|}
\hline & $\begin{array}{l}\text { US-agreement with MRI } \\
\text { volume at PSA-D categories }\end{array}$ & $\begin{array}{l}\text { US-MRI agreement at PSA-D } \\
\text { threshold } 0.15 \mathrm{ng} / \mathrm{mL}^{2}\end{array}$ & $\begin{array}{l}\text { US-MRI agreement at PSA-D } \\
\text { threshold 0.12 } \mathrm{ng}^{2} \mathrm{~mL}^{2}\end{array}$ \\
\hline $\begin{array}{l}\text { TA US-PSA } \\
\text { density }\end{array}$ & $53 / 64(83 \%)$ & $55 / 64(86 \%)$ & $61 / 64(95 \%)$ \\
\hline $\begin{array}{l}\text { TP US-PSA } \\
\text { density }\end{array}$ & $54 / 64(84 \%)$ & $56 / 64(88 \%)$ & $60 / 64(94 \%)$ \\
$\begin{array}{l}\text { TATP US- } \\
\begin{array}{l}\text { PSA } \\
\text { density }\end{array}\end{array}$ & $56 / 64(88 \%)$ & $56 / 64(88 \%)$ & $59 / 64(92 \%)$ \\
\hline $\begin{array}{l}\text { TP-TA US- } \\
\text { PSA } \\
\text { density }\end{array}$ & $54 / 64(84 \%)$ & $58 / 64(91 \%)$ & $59 / 64(92 \%)$ \\
\hline
\end{tabular}

Sensitivity, specificity, positive and negative predictive values were calculated for a matched decision to proceed to biopsy or not based on MRI PSAD and each U/S-derived PSAD at thresholds of $>0.15 \mathrm{ng} / \mathrm{mL}^{2}$ and $>0.12$ $\mathrm{ng} / \mathrm{mL}^{2}$, respectively (Tables 6,7 ). At a biopsy threshold of $>0.15 \mathrm{ng} / \mathrm{mL}^{2}$, sensitivity ranged between $74-78 \%$ and specificity $98 \%-100 \%$, PPV ranged between $96 \%-100 \%$ and NPV $88 \%-100 \%$, with the optimal results obtained by the US TA views, with a sensitivity of $100 \%$ and specificity $98 \%$. At a biopsy threshold of $>0.12 \mathrm{ng} / \mathrm{mL}^{2}$ sensitivity ranged between $81-100 \%$, specificity $85-100 \%$, PPV $86-100 \%$ and NPV between $83-100 \%$. Prioritising sensitivity, US TA had optimal sensitivity of $100 \%$, with a specificity of $85 \%$, and when prioritising specificity, US TP was optimal with sensitivity of $87 \%$ and specificity $100 \%$.

Table 6

Sensitivity, Specificity, Positive predictive value (PPV), Negative predictive value (NPV) between MRI-PSA density and each US PSA density for the decision to biopsy at PSA threshold $>0.15 \mathrm{ng} / \mathrm{mL}^{2}$

\begin{tabular}{|lllll|}
\hline & Sensitivity & Specificity & PPV & NPV \\
\hline TA US-PSA density & $100 \%$ & $98 \%$ & $96 \%$ & $100 \%$ \\
\hline TP US-PSA density & $91 \%$ & $100 \%$ & $100 \%$ & $95 \%$ \\
\hline TAVP US-PSA density & $74 \%$ & $100 \%$ & $100 \%$ & $88 \%$ \\
\hline TP-TA US-PSA density & $78 \%$ & $100 \%$ & $100 \%$ & $89 \%$ \\
\hline
\end{tabular}


Table 7

Sensitivity, Specificity, Positive predictive value (PPV), Negative predictive value (NPV) between MRI-PSA density and each US PSA

density for the decision to biopsy at PSA threshold $>0.12 \mathrm{ng} / \mathrm{mL}^{2}$

\begin{tabular}{|lllll|}
\hline & Sensitivity & Specificity & PPV & NPV \\
\hline TA US-PSA density & $100 \%$ & $85 \%$ & $86 \%$ & $100 \%$ \\
\hline TP US-PSA density & $87 \%$ & $100 \%$ & $100 \%$ & $89 \%$ \\
\hline TAVP US-PSA density & $84 \%$ & $100 \%$ & $100 \%$ & $87 \%$ \\
\hline TP-TA US-PSA density & $81 \%$ & $100 \%$ & $100 \%$ & $83 \%$ \\
\hline
\end{tabular}

\section{Discussion}

Our results demonstrate good overall agreement between surface-based US-derived and prostate volumes compared to MRI measurements with the greatest agreement shown between TA and TA/TP US views and MRI volume. We also demonstrate that risk-stratified decisions to biopsy based on US-derived PSAD thresholds strongly agree with MRI-derived measurements and therefore show potential for use as an initial point-of-care clinical test for either triaging MRI urgency at higher PSAD thresholds, or for avoiding the need for further investigations at lower thresholds. The results are encouraging as US is relatively inexpensive, readily available and non-invasive, and transabdominal scanning is a core sonographic skill [21].

Digital rectal examination estimated prostate size is known to lack accuracy, significantly underestimating prostate volume, particularly for larger glands, and cannot be used to derive measurements clinically [22]. MRI has been shown to be more accurate in volume assessment compared to transrectal US (TRUS) volumes estimated by urologists at the time of biopsy [23]. However, to our knowledge, no study has yet assessed PSAdensity derived from the less invasive transabdominal or transperineal US approaches, and when performed by experienced radiologists. Ozden, et al compared transabdominal US and TRUS sagittal, transverse and AP prostatic diameters, finding a strong correlation between the two techniques, with the greatest correlation demonstrated for sagittal views [24]. Likewise, Pate, et al found that prostate transabdominal volume showed good agreement with TRUS volume and could be used interchangeably when the prostate volume was less than $30 \mathrm{~mL}$, however, for larger glands, the authors recommend cross-sectional imaging for more accurate assessment [25]. Moreover, a recent systematic review comparing TRUS, CT and MRI prostatic volume measurements showed MRI as the optimal technique for deriving prostate volume [26]. Paterson, et al confirm these findings by demonstrating a better correlation of MRI prostate volume than TRUS using prostatectomy as the gold standard [23].

Despite its clinical utility, pre-biopsy prostate MRI is experience dependent $[27,28]$ and has ongoing limitations, leading to unnecessary, negative biopsies in around a third of patients [9-12], missing up to $20 \%$ of clinically significant cancers, and over-detecting insignificant lesions in around $10 \%$ of patients [13, 29-31]. However, MRIderived PSA-density, which adjusts the PSA levels proportionate to gland size is emerging as a useful clinical biomarker, with a threshold of $0.15 \mathrm{ng} / \mathrm{mL}^{2}$ suggested as a diagnostic cut-off that could be used to avoid help biopsy in selected patients [17]. MRI is an expensive and relatively scarce resource, therefore rationalizing use of such imaging would be of great clinical benefit. We investigated the agreement between MRI-PSA density and 
US-derived PSA density at the clinically meaningful threshold of $>0.15 \mathrm{ng} / \mathrm{ml}^{2}$ and subsequently the more conservative threshold of $>0.12 \mathrm{ng} / \mathrm{ml}^{2}$, also in part informed by the trend towards volume over-estimation with US. The results suggest that US-derived PSA density based decisions for biopsy match the MRI-derived decision most closely with US-TA views at a biopsy threshold of $>0.15$ and with either TP or TA views at a biopsy threshold of $>0.12$ depending whether sensitivity of specificity is maximised. Higher sensitivity is likely to be more of a clinical priority for decisions to biopsy, and this is provided by transabdominal views alone, likely due to the propensity to under-estimate gland volume and therefore "falsely increase" PSA density. However, transperineal views either alone or in combination with transabdominal views may be of benefit in certain clinical circumstances, because the views are relatively independent of the degree of bladder filling and the body habitus of the patient. Transabdominal US provides excellent views in the axial plane, however, sagittal measurements may be compromised by shadowing from the pubic bones at the level of the prostatic apex (Figure 2), conversely transperineal US sagittal views are unaffected by this when scanning across the symphysis pubis, however, the right-left axial measurements are significantly compromised by pubic bone shadowing, leading to an overall poorer performance.

Our study has some limitations, including the relatively small sample size. We chose to compare US-derived measured to the clinical standard of triplanar MRI measurements rather than MRI segmentation derived prostate volumes. This matches current PI-RADS guidelines recommendations, given that segmentation can be time consuming and software is not widely available [32]. Slight differences between triplanar and MRI volumetric measurements are not unexpected, as the ellipsoid formula assumes a regularly deformed sphere, however, asymmetrical BPH will lead to an over-estimation with this methodology, consistent with our findings [14, 33]. US image-acquisition quality is operator-dependent and, in particular for the more specialist technique of transperineal acquisition, performed by uro-radiologists; this may not be available in all healthcare settings, and may therefore affect the generalisability of our results. More studies are needed to evaluate whether these results can be replicated by non-specialist radiologists or sonographers, and whether there is potential for future translation into a primary care setting. Despite the prospective study design, the results of the study did not influence clinical decision-making. It should also be noted that in the clinical setting PSAD is used alongside MRI findings and therefore may be insufficient alone to avoid MRI, and prospective studies would be required to evaluate US-derived PSAD measurements against clinical outcomes and the decision to biopsy. It may also be possible to combine US-PSAD with blood or urine biomarkers, such as PHI to further augment a risk-stratified clinical decision process [34]. US did not always provide accurate measurements, however, we found this was often predictable, for instance in patients undergoing a prior TURP procedure, or having a high BMI.

In conclusion, we demonstrate that surface-based US-derived PSA density has good agreement with MRI-derived values and with the clinical risk-stratified decision for biopsy, and offers potential for supplementing clinical decision-making to triage patients to undergo urgent MR imaging or even avoid unnecessary MRI in selected patients.

\section{Declarations}

\section{Acknowledgements}

This research was supported by the National Institute of Health Research Cambridge Biomedical Research Centre (BRC-1215-20014). The views expressed are those of the author(s) and not necessarily those of the NIHR 
or the Department of Health and Social Care. The authors also acknowledge support from Cancer Research UK (Cambridge Imaging Centre grant number C197/A16465), the Engineering and Physical Sciences Research Council Imaging Centre in Cambridge and Manchester and the Cambridge Experimental Cancer Medicine Centre.

\section{References}

1. Siegel RL, Miller KD, Jemal A. Cancer Statistics, 2017. CA Cancer J Clin. 2017;67(1):7-30

2. Maddams J, Utley M, Møller H. Projections of cancer prevalence in the United Kingdom, 2010-2040. Br J Cancer. 2012; 107(7):1195-1202

3. Stamey TA, Caldwell M, McNeal JE, et al (2004) The prostate specific antigen era in the United States is over for prostate cancer: What happened in the last 20 years? J Urol 172:1297-1301

4. Schröder FH, van der Cruijsen-Koeter I, de Koning HJ, Vis AN, Hoedemaeker RF, Kranse R. Prostate cancer detection at low prostate specific antigen. J Urol. 2000;163(3):806-12

5. Adhyam M., Gupta A.K. A Review on the Clinical Utility of PSA in Cancer Prostate Indian J Surg Oncol. 2012; 3(2): $120-129$

6. US Preventive Services Task Force. Final Recommendation Statement: Prostate Cancer: Screening - US Preventive Services Task Force.

https://www.uspreventiveservicestaskforce.org/Page/Document/RecommendationStatementFinal/prostatecancer-screening1\#table-of-contents [Accessed 03 August 2021]

7. Mottet N, van den Bergh RCN, Briers E, et al. EAU - ESTRO - ESUR - SIOG Guidelines on Prostate Cancer 2019. In: European Association of Urology Guidelines. 2019 Edition. Vol Edn. presented at the EAU Annual Congress Barcelona 2019. Arnhem, The Netherlands: European Association of Urology Guidelines Office; 2019

8. Bjurlin MA, Carroll PR, Eggener S, et al. Update of the Standard Operating Procedure on the Use of Multiparametric Magnetic Resonance Imaging for the Diagnosis, Staging and Management of Prostate Cancer. J Urol. 2020; 203(4):706-712

9. van der Leest M, Cornel E, Israel B, et al. Head-to-head comparison of transrectal ultrasound-guided prostate biopsy versus multiparametric prostate resonance imaging with subsequent magnetic resonance-guided biopsy in biopsy-naïve men with elevated prostate-specific antigen: a large prospective multicenter clinical study. Eur Urol. 2019; 75(4):570-578 [7]

10. Barrett T, Slough RA, Sushentsev N, et al. 3-year experience of a dedicated prostate mpMRI pre-biopsy programme and effect on timed cancer diagnostic pathways. Clin Radiol. 2019; 74(11):894.e1-894.e9

11. Ahmed HU, El-Shater Bosaily A, Brown LC, et al; PROMIS study group. Diagnostic accuracy of multiparametric MRI and TRUS biopsy in prostate cancer (PROMIS): a paired validating confirmatory study. Lancet. 2017; 389(10071):815-822

12. Kasivisvanathan V, Rannikko AS, Borghi M, et al. MRI-targeted or standard biopsy for prostate-cancer diagnosis. New England Journal of Medicine. 2018;378(19):1767-77.

13. Sushentsev N, Caglic I, Sala E, et al. The effect of capped biparametric magnetic resonance imaging slots on weekly prostate cancer imaging workload. Br J Radiol. 2020 Apr;93(1108):20190929.

14. Hansen NL, Barrett T, Koo B, et al. The influence of prostate-specific antigen density on positive and negative predictive values of multiparametric magnetic resonance imaging to detect Gleason score 7-10 prostate 
cancer in a repeat biopsy setting. BJU Int. 2017;119(5):724-30.

15. Distler FA, Radtke JP, Bonekamp D, et al. The Value of PSA Density in Combination with PI-RADS ${ }^{\mathrm{TM}}$ for the Accuracy of Prostate Cancer Prediction. J Urol. 2017;198(3):575-582

16. Boesen L, Nørgaard N, Løgager V, et al. Prebiopsy Biparametric Magnetic Resonance Imaging Combined with Prostate-specific Antigen Density in Detecting and Ruling out Gleason 7-10 Prostate Cancer in Biopsynaïve Men. Eur Urol Oncol. 2019;2(3):311-319

17. Brizmohun Appayya M, Adshead J, et al. National implementation of multi-parametric magnetic resonance imaging for prostate cancer detection-recommendations from a UK consensus meeting. BJU international. 2018;122(1):13-25.

18. Jayawant M.N. Measures of Interrater Agreement. J Thorac Oncol. 2015; 6 (1): 6-7.

19. Koo TK, Li MY. A guideline of selecting and reporting intraclass correlation coefficients for reliability research. J Chiropr Med. 2016; 15(2):155-63

20. Khoo CC, Eldred-Evans D, Peters M, Bertoncelli Tanaka M, et al. Likert vs PI-RADS v2: a comparison of two radiological scoring systems for detection of clinically significant prostate cancer. BJU Int. 2020; 125(1):4955

21. Lee JS, Chung BH. Transrectal ultrasound versus magnetic resonance imaging in the estimation of prostate volume as compared with radical prostatectomy specimens. Urol Int. 2007;78(4):323-7

22. Roehrborn CG. Accurate determination of prostate size via digital rectal examination and transrectal ultrasound. Urology. 1998;51(4A Suppl):19-22

23. Paterson NR, Lavallée LT, Nguyen LN, et al. Prostate volume estimations using magnetic resonance imaging and transrectal ultrasound compared to radical prostatectomy specimens. Canadian Urological Association Journal. 2016;10(7-8):264.

24. Ozden E, Göğüş C, Kiliç O, Yaman O, Ozdiler E. Analysis of suprapubic and transrectal measurements in assessment of prostate dimensions and volume: is transrectal ultrasonography really necessary for prostate measurements? Urol J. 2009;6(3):208-13

25. Pate WR, Garg N, Wang LB, Wason SE, Barbosa PV. Comparison of Transabdominal and Transrectal Ultrasound for Sizing of the Prostate. Urology. 2020; 141:125-129.

26. Christie DRH, Sharpley CF. How Accurately Can Prostate Gland Imaging Measure the Prostate Gland Volume? Results of a Systematic Review. Prostate Cancer. 2019; 2019:6932572

27. de Rooij M, Israël B, Tummers M, et al. ESUR/ESUI consensus statements on multi-parametric MRI for the detection of clinically significant prostate cancer: quality requirements for image acquisition, interpretation and radiologists' training. Eur Radiol. 2020; 30(10):5404-5416

28. Barrett T, Padhani AR, Patel A, et al. Certification in reporting multiparametric magnetic resonance imaging of the prostate: recommendations of a UK consensus meeting. BJU Int. 2021;127(3):304-306

29. Schoots IG, Roobol MJ. Multivariate risk prediction tools including MRI for individualized biopsy decision in prostate cancer diagnosis: current status and future directions. World J Urol. 2020;38(3):517-529

30. Drost FH, Osses DF, Nieboer D, Steyerberg EW, Bangma CH, Roobol MJ, Schoots IG. Prostate MRI, with or without MRI-targeted biopsy, and systematic biopsy for detecting prostate cancer. Cochrane Database Syst Rev. 2019;4(4):CD012663 
31. Serrao EM, Barrett T, Wadhwa K, et al. Investigating the ability of multiparametric MRI to exclude significant prostate cancer prior to transperineal biopsy. Can Urol Assoc J. 2015;9(11-12):E853-8

32. Barrett T, Rajesh A, Rosenkrantz AB, Choyke PL, Turkbey B. PI-RADS version 2.1: one small step for prostate MRI. Clin Radiol. 2019;74(11):841-852

33. Turkbey B, Fotin SV, Huang RJ, et al. Fully automated prostate segmentation on MRI: comparison with manual segmentation methods and specimen volumes. AJR Am J Roentgenol. 2013;201(5):W720-9

34. Gnanapragasam VJ, Burling K, George A, et al. The Prostate Health Index adds predictive value to multiparametric MRI in detecting significant prostate cancers in a repeat biopsy population. Sci Rep. 2016; 6:35364

\section{Figures}



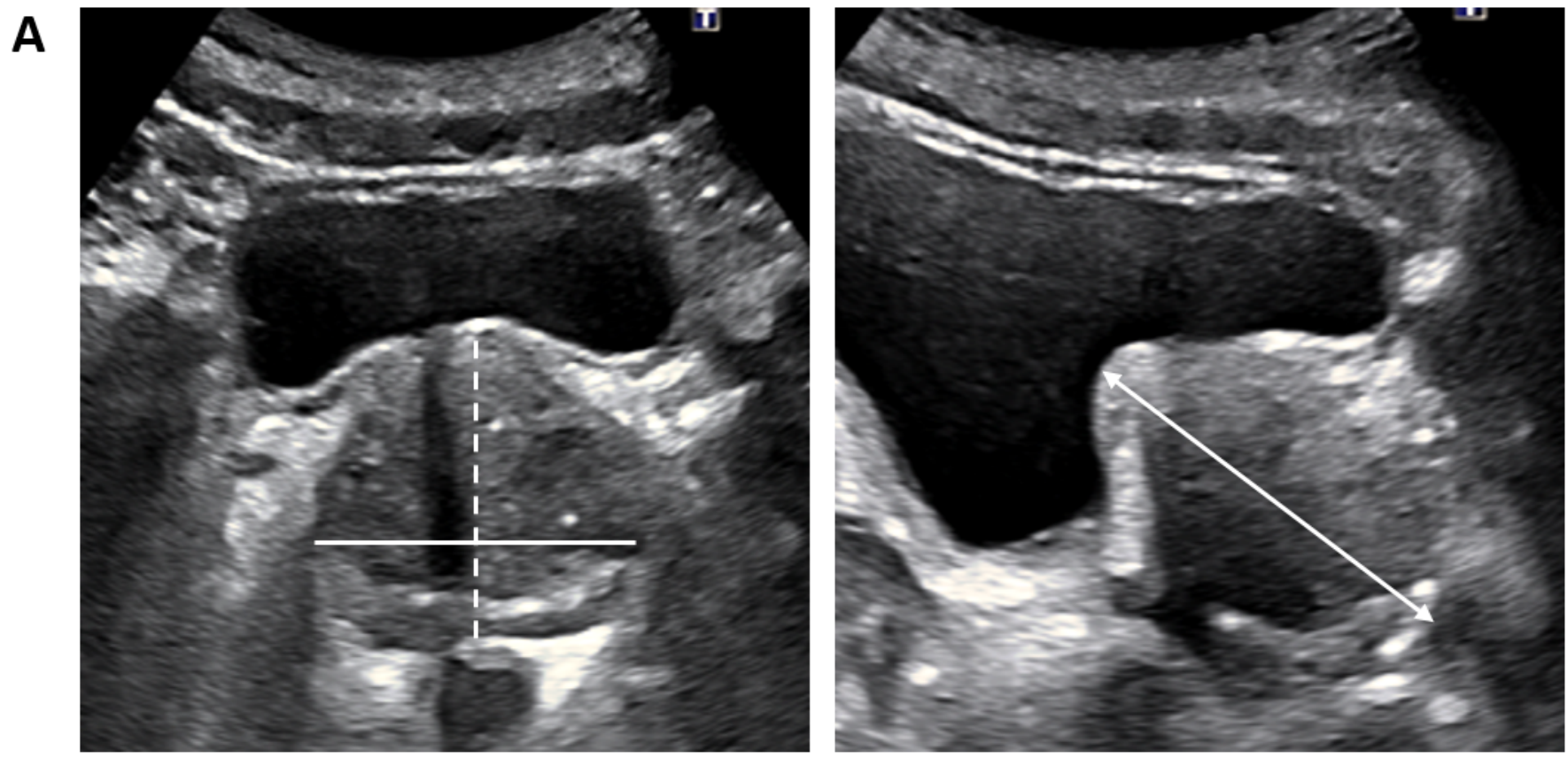

B
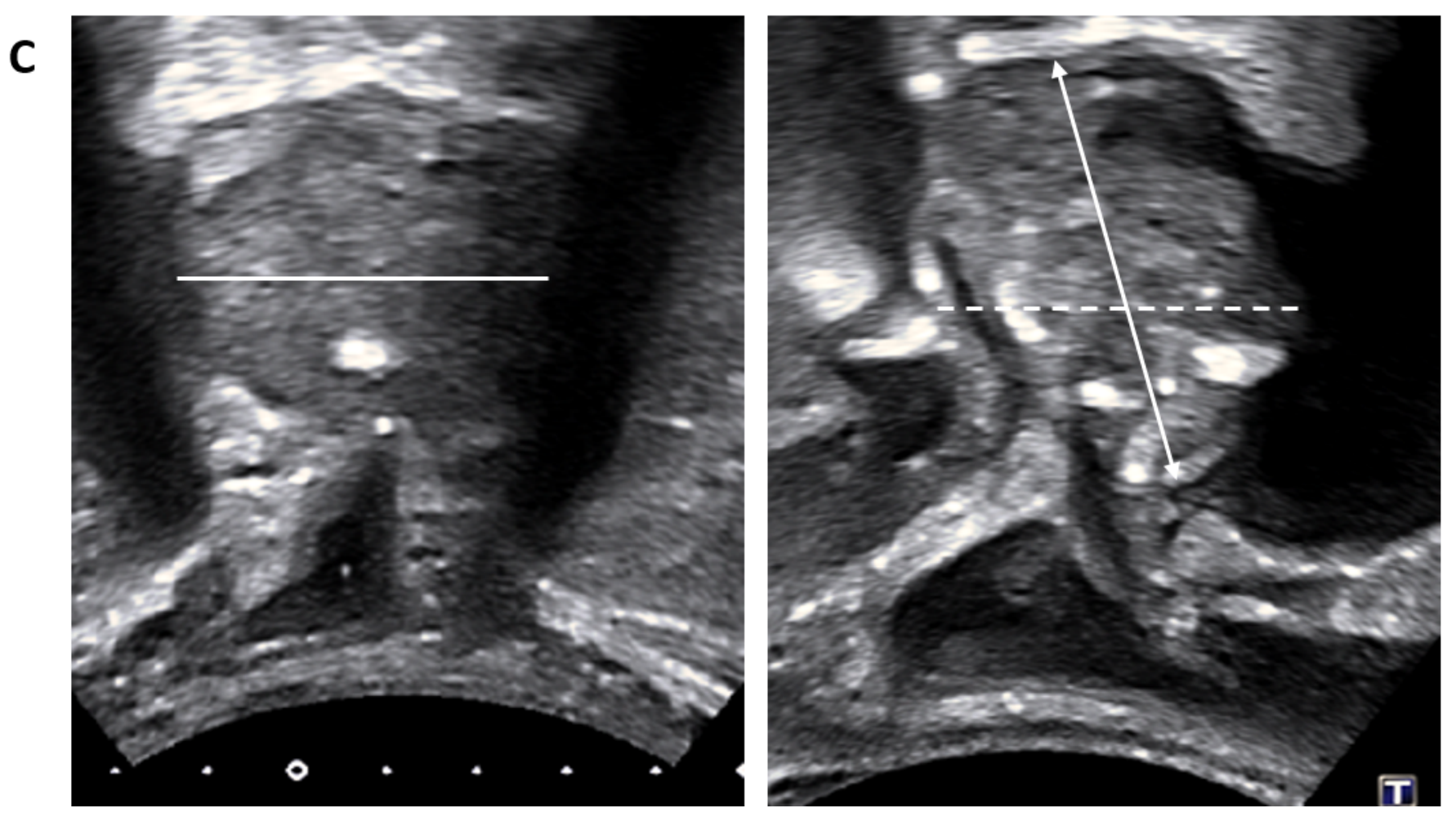

\section{Figure 1}

Ultrasound views obtained. Transabdominal views in the transverse/axial (A) and longitudinal/sagittal (B) planes. Transperineal views in the longitudinal/sagittal (C) and transverse/coronal planes (D). Lines represent "Axial 1" measurements (right to left), dashed lines represent "Axial 2" measurements (anterior-posterior), arrowed lines represent Sagittal measurements. 
A
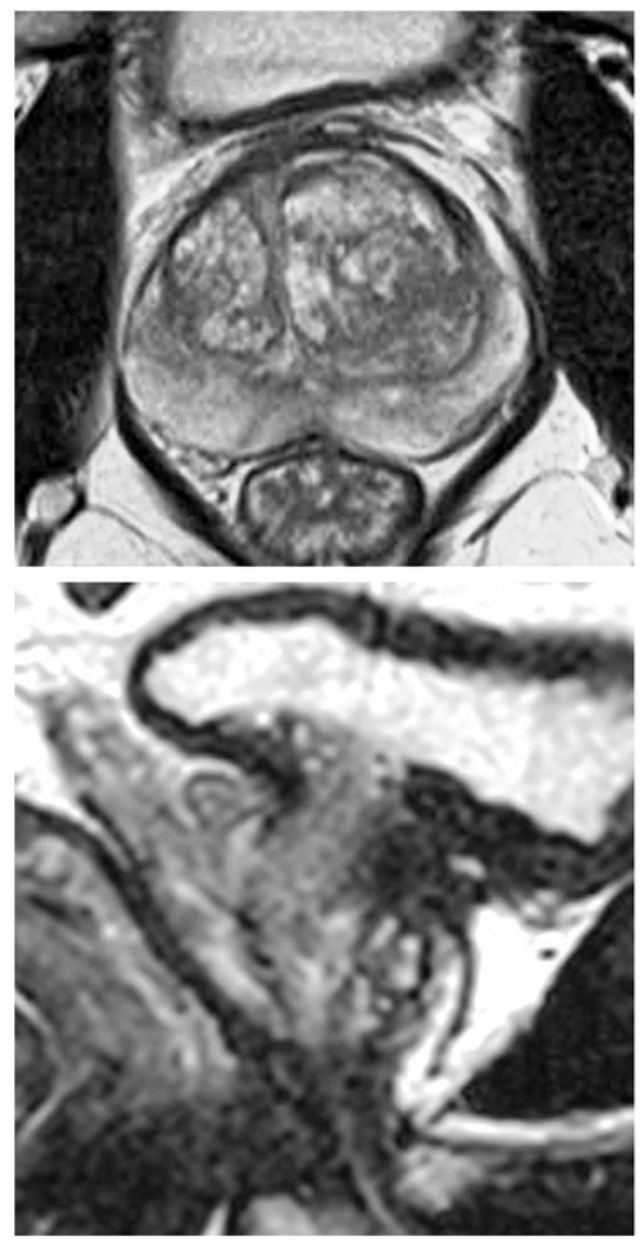

D
B
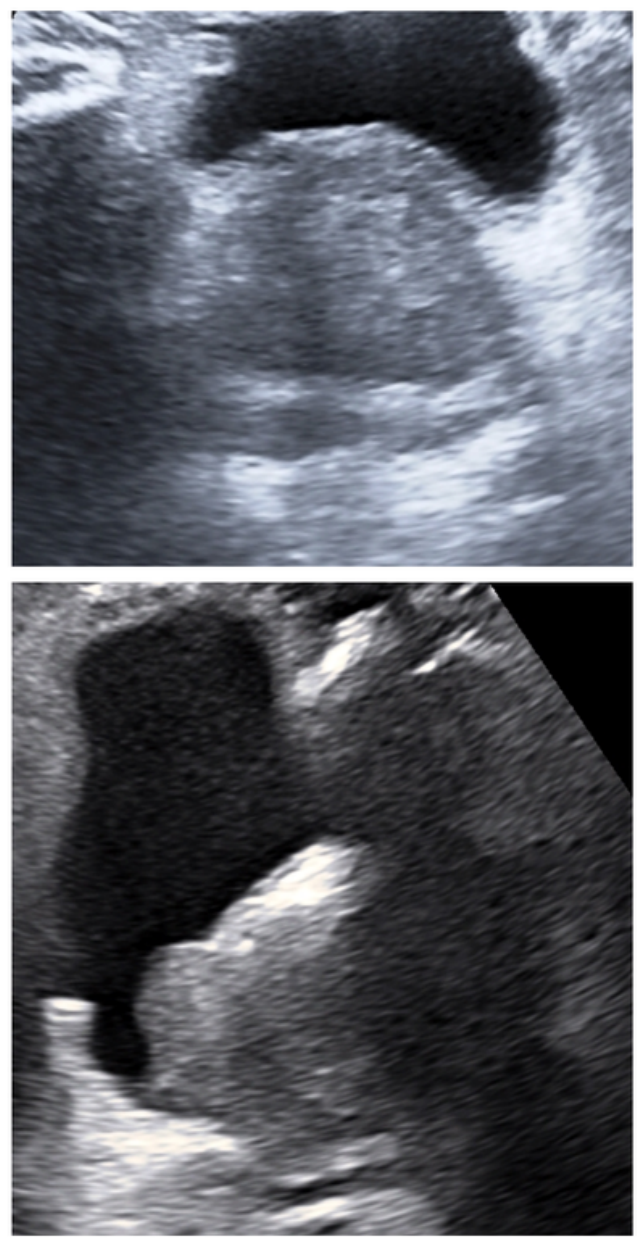

E
C
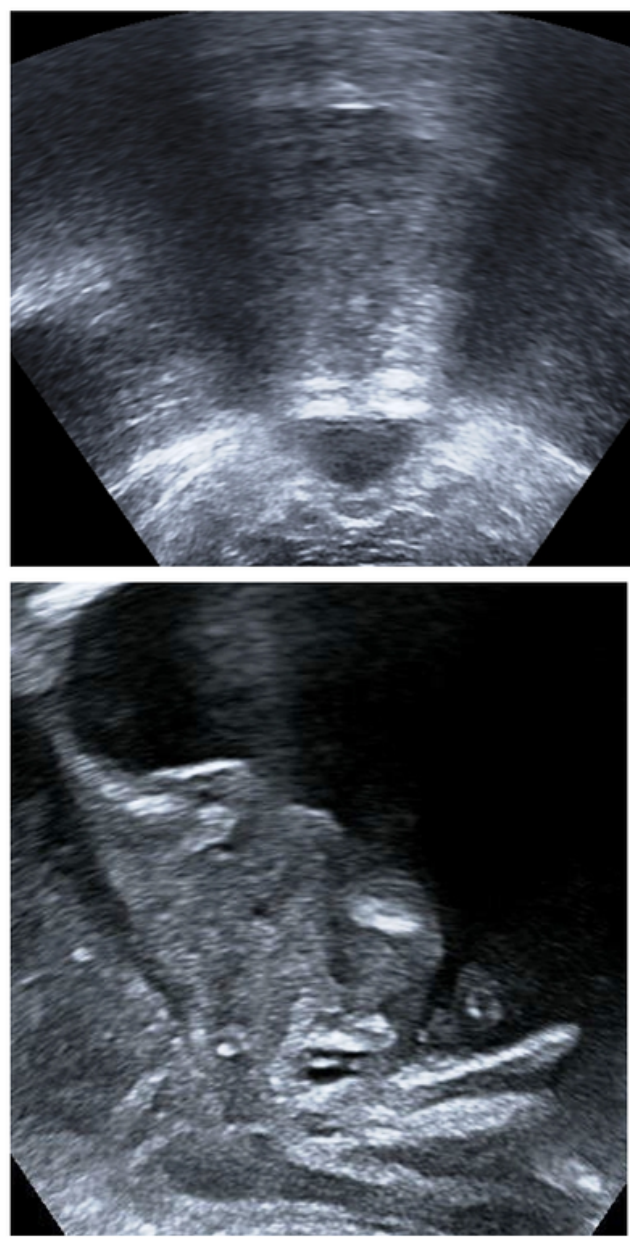

$\mathbf{F}$

\section{Figure 2}

Example Transabdominal (TA) and Transperineal (TP) US views. Axial (A) and sagittal (D) MR images. B, E: TA ultrasound views from the same patient in the axial (B) and sagittal planes (E). C, F: TP ultrasound views in the axial (C) and sagittal planes (F). The sagittal TA US view (E) has shadowing from the pubis bone overlying the prostatic apex, which may affect measurement accuracy, conversely shadowing affects the axial view of TP imaging (C). 
A

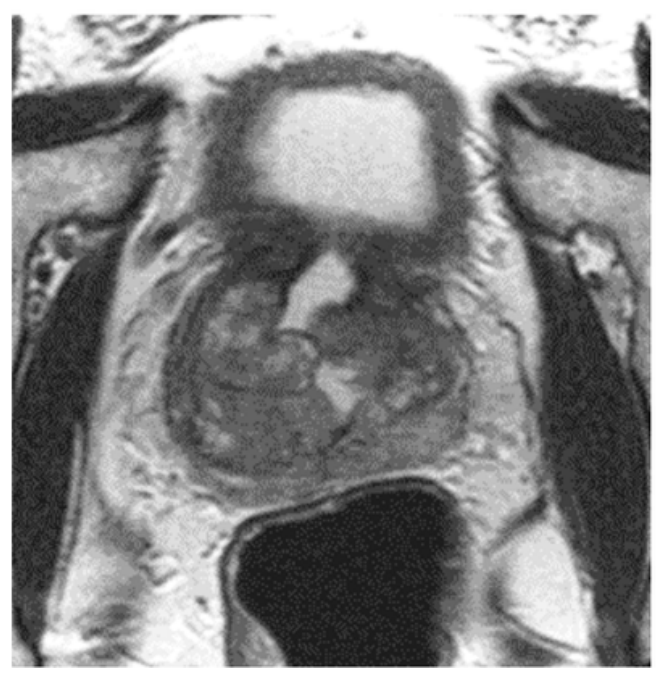

B

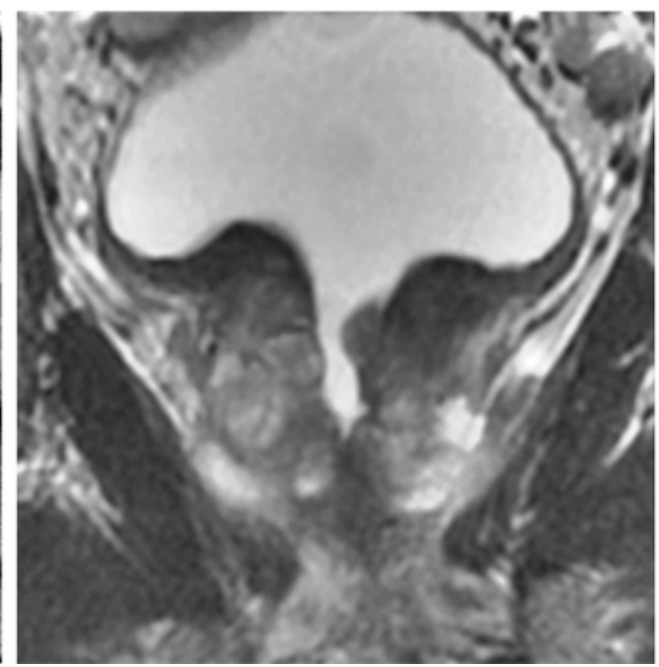

C

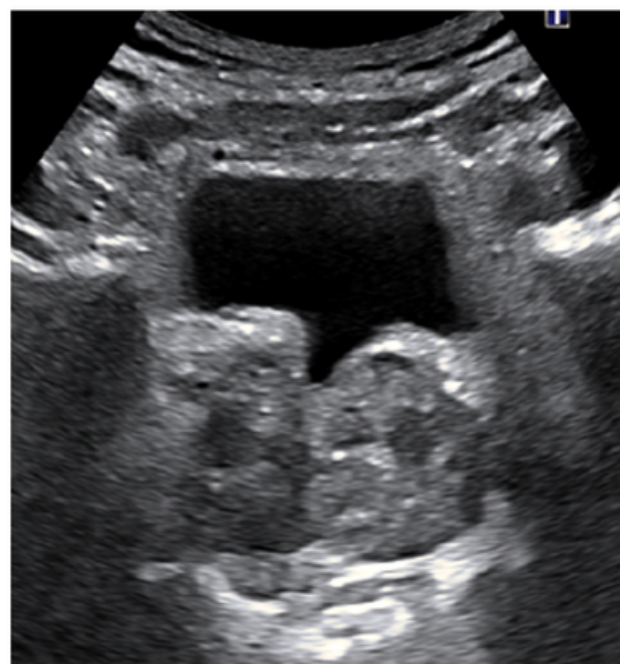

Figure 3

TURP defect affects measurements. Axial (A) and coronal (B) T2-weighted images show TURP defect at the base of the gland, with corresponding transverse transabdominal US image (C). MRI triplanar and segmentation volumes will differ, and sagittal US measurements will vary depending if the gland is measured in the midline position, or more laterally.

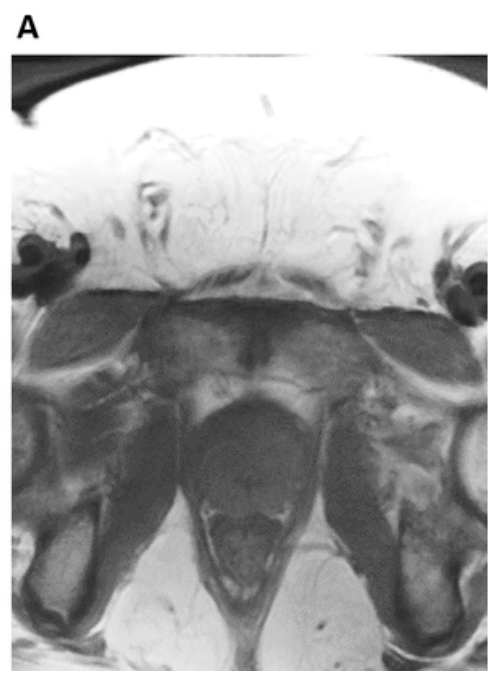

B

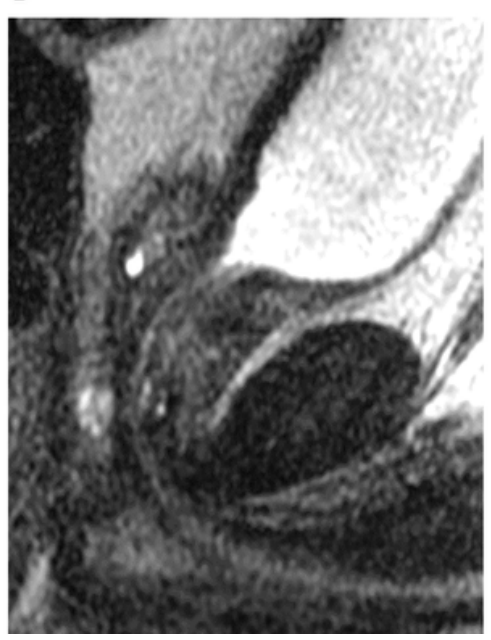

C

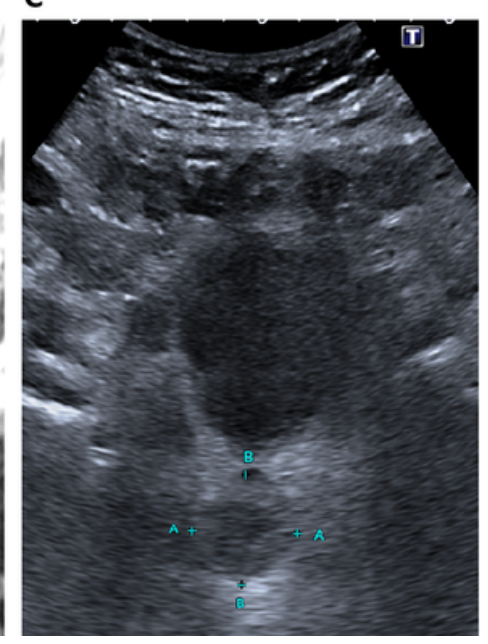

D

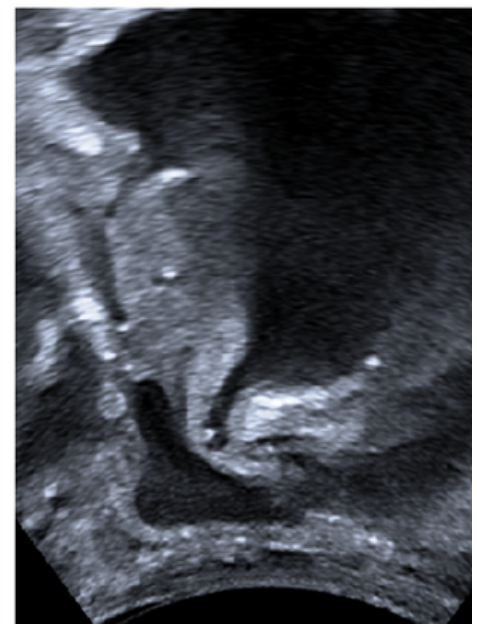

\section{Figure 4}

Limited views due to patient body habitus. MRI Axial T1-weighted image (A) shows depth of prostate; corresponding sagittal T2-weighted image of the prostate (B). C: Transabdominal transverse imaging is limited (calipers outline prostate). D: Transperineal sagittal views more clearly demonstrate the prostate. 